\title{
Eristalis (Diptera: Syrphidae) Flower Flies are Potential Non-host Vectors of the Common Trypanosome Bee Parasite, Crithidia Bombi
}

Abby E. Davis ( $\square$ aed236@cornell.edu )

Cornell University

Kaitlin R. Deutsch

Cornell University

Alondra M. Torres

Cornell University

Mesly J. Mata Loya

Cornell University

Lauren Cody

Cornell University

\section{Emma Harte}

Cornell University

\section{David Sossa}

Cornell University

Paige A. Muñiz

Cornell University

Wee Hao Ng

Cornell University

Scott H. McArt

Cornell University

\section{Research Article}

Keywords: Flowers , transmission platforms, parasites, bee health,Crithidia bombi, Eristalis tenax

Posted Date: May 27th, 2021

DOI: https://doi.org/10.21203/rs.3.rs-556537/v1

License: (c) (1) This work is licensed under a Creative Commons Attribution 4.0 International License.

Read Full License 
Version of Record: A version of this preprint was published at Scientific Reports on August 4th, 2021. See the published version at https://doi.org/10.1038/s41598-021-95323-w. 


\section{Abstract}

Flowers can be transmission platforms for parasites that impact bee health, yet bees share floral resources with other pollinator taxa, such as flies, that could be hosts or non-host vectors (i.e., mechanical vectors) of parasites. Here, we assessed whether the fecal-orally transmitted gut parasite of bees, Crithidia bombi, can infect Eristalis tenax flower flies. We also investigated the potential for two confirmed solitary bee hosts of $C$. bombi, Osmia lignaria and Megachile rotundata, as well as two flower fly species, Eristalis arbustorum and E. tenax, to transmit the parasite at flowers. We found that $C$. bombi did not replicate (i.e., cause an active infection) in $E$. tenax flies. However, $93 \%$ of inoculated flies defecated live $C$. bombi in their first fecal event, and all contaminated fecal events contained $C$. bombi at concentrations sufficient to infect bumble bees. Flies and bees defecated inside the corolla (flower) more frequently than other plant locations, and flies defecated at volumes comparable to or greater than bees. Our results demonstrate that Eristalis flower flies are not hosts of $C$. bombi, but they may be mechanical vectors of this parasite at flowers. Thus, flower flies may amplify or dilute $C$. bombi in bee communities.

\section{Introduction}

Recent analysis of long-term sampling data and biological records have shown that globally, wild insect pollinators, including solitary bees and flies, are experiencing population declines and range contractions 1-4. Parasites are key drivers of pollinator health and are associated with declines of several pollinator species ${ }^{5-7}$. This fact is concerning for both conservation and economic reasons; pollination services are valued at more than $\$ 170$ billion/year globally ${ }^{8}$, solitary bees and flies perform a large proportion of the services ${ }^{9-12}$, and agricultural dependence on pollinators continues to increase each year ${ }^{13}$.

Most information about pollinator parasites is known from social bees $7,14,15$. Honey bees and bumble bees were previously thought to be the only host of certain viruses, microsporidians and trypanosomes, but recent studies have found many of these same parasites are present in and can infect wild solitary bee species, too ${ }^{16-22}$. The host range of these parasites, however, is understudied, and limited studies have assessed the incidence and infectivity of bee parasites in non-bee pollinators.

Evison et al ${ }^{23}$ reported high prevalence of Wolbachia bacteria and Ascosphera fungi, and low prevalence of microsporidian fungi ${ }^{23}$, among wild-caught flower flies and bees. Additionally, Bailes et al ${ }^{24}$ found high viral titres of two honey bee viruses (Sacbrood Virus and Black Queen Cell Virus) in wild-caught Eristalis (Diptera: Syrphidae) flower flies ${ }^{24}$. The nucleotide sequences of these viruses were $87-100 \%$ similar to those found in co-foraging honey bees, suggesting the viruses were not strains unique to flies and possibly being shared between the two taxa. Similarly, Brettell et al. ${ }^{25}$ also found bee-associated viruses in wild-caught flower flies after deep sequencing ${ }^{25}$. While these studies suggest many bee parasites may be broad, multi-host parasites, they do not show whether infection (active replication of the parasites) is occurring in non-bee pollinators, nor how transmission occurs. 
The trypanosome gut parasite Crithidia bombi lacks cell-specific host requirements compared to intracellular parasites such as microsporidian Nosema spp. ${ }^{26,27,28}$, has been found in a wide variety of solitary and social bees $15,21,22$, and despite historically being considered a "bumble bee parasite," has recently been found to replicate in two species of solitary bees, the alfalfa leafcutter bee, Megachile rotundata, and blue orchard bee, Osmia lignaria ${ }^{21,22}$. The rather broad host requirements of $C$. bombi indicate this parasite may be able to infect, or pass through, the guts of many different flower-visiting insects. However, beyond the studies mentioned above, it is unknown if other flower-visiting insects such as flower flies (Diptera: Syrphidae) can act as hosts of $C$. bombi. This is important since flower flies occupy a similar ecological niche as bees as a result of their similar morphology, behaviors and foraging habits ${ }^{29,30}$.

In addition to host competence, epidemiology of multi-host parasites is also shaped by the environment. One particularly relevant factor to pollinator parasites are flowers - key hubs of transmission. Graystock, Goulson \& Hughes ${ }^{7}$ experimentally demonstrated that infected honey bees can transmit common, fecaloral bee parasites including $C$. bombi to susceptible bumble bees (and vice versa) simply by foraging on the same flowers ${ }^{31}$. Furthermore, a recent study that screened nearly 3,000 flowers in nature found one in eleven flowers harbored pollinator parasites, including $C$. bombi ${ }^{15}$. Multiple factors such as landscape simplification ${ }^{32}$, presence of managed social bees ${ }^{18}$, floral traits ${ }^{33,34}$, and location of parasites on flowers ${ }^{35}$ can influence the prevalence and likelihood of transmission of pollinator parasites at flowers. Flies may contribute to transmission by mechanically spreading parasites from contaminated flowers, potentially redistributing the parasites on flowers during defecation and therefore creating more floral transmission hotspots. However, whether flies can act as mechanical vectors and transmit pollinator parasites at flowers, at quantities that can infect bees, is unknown. In addition, how flies compare to bees as parasite transmitters at flowers is also unknown.

As adult Eristalis flies visit the same floral resources as bees, they, too, can encounter, ingest and potentially become infected by common fecal-orally transmitted "bee" parasites. Therefore, we assessed: 1) whether the common, fecal-orally transmitted "bee" parasite, $C$. bombi, could infect the cosmopolitan European drone fly, Eristalis tenax, 2) whether the quantity of viable $C$. bombi cells defecated by $E$. tenax would be sufficient to infect a common host of $C$. bombi, Bombus impatiens, and 3) whether two species of Eristalis flies ( $E$. arbustorum and E. tenax) as well as two megachilid bee hosts (M. rotundata and $O$. lignara) defecate on flowers and therefore could potentially transmit $C$. bombi at flowers.

\section{Results}

\subsection{Evaluating whether the European drone fly, Eristalis tenax, is a host or non-host vector of Crithidia bombi 3.1.1 Inoculation of Eristalis tenax with Crithidia bombi}


E. tenax flies were inoculated with $C$. bombi and both the first defecation event and gut were screened for the parasite. C. bombi was never found in the gut of the flies 10 days post-inoculation (Table 1). However, all flies defecated within 5 hours of the start of the experiment and the first fecal event from $93 \%$ of the inoculated flies $(n=30)$ contained live $C$. bombi (Table 1). The average amount of live $C$. bombi in these first fecal events were 239 parasites (95\% Cl: 174.4-362.7 parasites; Fig. 1a). One fecal event contained a total of 1,080 cells, which was the greatest concentration and equated to roughly one-third of the inoculum fed to the flies. There was no significant difference in $C$. bombiload between males vs. females $\left(\mathrm{LRT}, \chi^{2}{ }_{1}=0.19, \mathrm{p}=0.66\right.$ ) and no $C$. bombi were found in the first defecation events of the control flies ( $n$ $=30$; Table 1). The average first fecal volume among the inoculated flies was $1.21 \mu \mathrm{l}(95 \% \mathrm{Cl}$ : $1.03-1.39$ $\mu \mathrm{l}$; Supplementary Figure S1). Together, these results indicate that $C$. bombi cells can survive passage through fly digestive tracts, although they do not cause active infections in the flies.

\subsubsection{Response of Bombus impatiens to varying doses of Crithidia bombi}

Bumble bees (B. impatiens workers) from two colonies were inoculated with varying doses (between 12 to 25,000 cells) of $C$. bombi as shown in Fig. 1 b. These doses were chosen to include the realistic range of $C$. bombi in flower fly feces (shown above) and beyond. Infection probability increased with dose, and the slope of the relationship was colony-dependent (dose-colony interaction: $\operatorname{LR}=9.6, p=0.002$, Supplementary Figure S2a). Smaller bees were also more likely to become infected ( $L R=14, p<0.001)$. Among the infected bees, infection intensity increased with dose $\left(t_{69}=2.8, p=0.007\right)$, was also colonydependent $\left(t_{69}=-2.122, p=0.037\right.$, Figure S2b), and smaller bees had higher infection intensities $\left(t_{69}=\right.$ $-2.8, p=0.006$ ). Conditional (pseudo-) $R^{2}$ of the models were 0.43 for infection probability, and 0.27 for infection intensity.

We generated dose-response curves for the two responses by marginalizing across colony and bee size (see Fig. 1b for infection probability, and Supplementary Figure S3 for infection intensity). Marginal (pseudo-) $R^{2}$, based on dose alone, was 0.27 for infection probability and 0.15 for infection intensity. Generalized linear models assume linear relationships between the link function and predictor. For each response and each colony, we did not find substantially stronger support for more flexible monotonic additive models (infection probability: $\triangle \mathrm{AIC}=-3 \times 10^{-4}$ (colony 1 ), 1.2 (colony 2); infection intensity: $\triangle \mathrm{AIC}=0.016$ (colony 1$), 1.3$ (colony 2 ), suggesting that the linear relationships were adequate at capturing the shape of the dose-response curves. 
Table 1

Proportion of inoculated and control Eristalis tenax flies with live Crithidia bombi cells in first fecal events (day 1 ) and guts 10 days post-inoculation.

\begin{tabular}{|lll} 
Treatment & Live $C$. bombi in fly feces (day 1$)$ & $\begin{array}{l}\text { Live } C . \\
\text { (day } 10)\end{array}$ \\
\hline Control & $0 / 30$ & $0 / 30$ \\
\hline Inoculated & $28 / 30$ & $0 / 30$ \\
\hline
\end{tabular}

We found that all treatments but the 12-cell dosage of $C$. bombi could infect bumble bees. The lowest infective dose ( 24 cells) had a $14 \%$ likelihood of bee infection and the highest dose (25,000 cells) had an $85 \%$ likelihood of bee infection. When we mapped the levels of $C$. bombi found in inoculated flower fly feces to the likelihood of infection using the dose-response curve, we found that all flower fly feces containing viable $C$. bombi could potentially infect $B$. impatiens. The lowest quantity of $C$. bombi parasites defecated by a fly was 60 cells, which had a $24 \%$ likelihood of bee infection (Fig. 1b). The highest quantity of $C$. bombi parasites defecated was 1,080 cells, corresponding to a $54 \%$ likelihood of infection if consumed by $B$. impatiens. The mean quantity of $C$. bombi parasites ( 239 cells) corresponds to a $35 \%$ likelihood of infection if consumed by B. impatiens (Fig. 1b).

\subsection{Vectoring potential of two bee species, Osmia lignaria and Megachile rotundata, and two Eristalis fly species, $\mathrm{E}$. arbustorum and $\mathrm{E}$. tenax}

\subsubsection{Fecal volumes and defecation frequency}

All pollinators were placed in individual cages lined with filter paper and fed ad libitum sucrose solution with fluorescent powder to measure fecal volumes and defecation frequency in a 24-hour period. When comparing fecal volumes between different species and sex using the filter paper data only (which used identical methodology for all pollinators), we found that the species-sex interaction was significant $\left(F_{(3 \text {, }}\right.$ $457)=10, p<0.001$; Fig. 2), so main effects were not tested in accordance to principle of marginality. Based on post-hoc pairwise contrasts (Supplementary Table S1), we found that for both sexes, E. tenax had significantly larger fecal volumes than all three other pollinator species, while $E$. arbustorum and $O$. lignaria both had larger fecal volumes than M. rotundata (Fig. 2). Within each species, only E. tenax showed a significant difference between sexes, with females having larger fecal volumes than males.

For E. tenax only, comparing the fecal volumes from the two methods (collected from microcentrifuge tubes in the inoculation experiment vs. estimated from filter paper spot diameters), we found that the method-sex interaction was marginally significant $\left(F_{(1,126)}=3.9, p=0.052\right)$, while both the main effects of method and sex were significant (method: $F_{(1,127)}=6.0, p=0.016$; sex: $F_{(1,127)}=22, p<0.001$ ). In particular, we found that fecal volumes collected from the microcentrifuge tubes were about $25 \%(95 \% \mathrm{Cl}$ : 
4.6\%-50\%) larger than fecal volumes estimated using standard curves from filter paper spot diameters (Supplementary Figure S4). Nonetheless, since the interaction was not significant, this means that any inference about fecal volume differences between groups should not be affected by the choice of methods.

E. tenax flies defecated more frequently than $E$. arbustorum flies in a 24-hour period $\left(F_{(1,137)}=85, p<\right.$ 0.001 ; Fig. 3). Uninfected E. arbustorum flies defecated, on average, 14 times in a 24-hour period and uninfected $E$. tenax flies defecated, on average, 32 times in a 24-hour period. Neither the species-sex interaction nor sex were important predictors of defecation frequency of $E$. arbustorum and $E$. tenax flies $\left(F_{(1,136)}=0.24, p=0.63 ; F_{(1,137)}=2.2, p=0.14\right)$.

\subsubsection{Defecation patterns on a shared floral resource}

Defecation events were recorded on Solidago in all cage trials with $E$. tenax flies ( $n=20$ trials) and $O$. lignaria bees ( $n=10$ trials), while E. arbustorum defecated on Solidago in only 8 of 10 trials. Both $E$. arbustorum and $O$. lignaria defecated on all locations of Solidago, while E. tenax defecated on all locations but the bract (Fig. 4). The interaction between pollinator species and plant location was not significant (LRT, $\chi^{2}{ }_{10}=16, p=0.093$ ), while both species and location main effects were significant (LRT, $\left.\chi_{2}^{2}=88, p<0.001 ; \chi^{2}{ }_{5}=90, p<0.001\right)$. Post-hoc tests indicate that $E$. tenax defecated on Solidago more often than $E$. arbustorum ( $Z$-test, $Z_{(\mathrm{Inf})}=2.778, p=0.015$ ), and $O$. lignaria defecated on Solidago more often than E. tenax and E. arbustorum, respectively (post-hoc Z-test, $Z_{(\text {Inf) }}=-7.261, p<0.001 ; Z_{(\operatorname{Inf})}=$ $-7.446, p=<0.001)$.

We found that the inside of the corolla was defecated on most often (compared to the outside of the corolla, sepal, bract, leaf and stem) respectively ( $p<0.001$ in each case; Fig. 4$)$. In addition, the leaf was defecated on more frequently than the bract (post-hoc $Z$-test, $Z_{(\operatorname{lnf})}=-3.542, p=0.005$ ).

\section{Discussion}

In this study, we found that $C$. bombi did not replicate and cause an active infection in E. tenax flies. However, $93 \%$ of inoculated flies defecated live $C$. bombi in their first fecal event, often at levels capable of infecting bumble bees. In addition, we show that Eristalis arbustorum and E. tenax both defecate comparable or larger volumes, respectively, of feces compared to Megachile rotundata and Osmia lignaria, two solitary bees that are recently confirmed hosts of $C$. bombi ${ }^{21,22}$. Furthermore, $E$. tenax and $E$. arbustorum are both shown to defecate on flowers, which are indirect transmission routes for $C$. bombi 31,35,36. Taken together, these results indicate that while Eristalis flower flies are not hosts of $C$. bombi, they can potentially be non-host vectors (i.e., mechanical vectors) that contribute to community-wide transmission of this multi-host parasite.

Infected bumble bees are known to shed similar parasite loads to the inoculum we fed to $E$. tenax flies ${ }^{37-}$ 39. In fact, heavily infected bumble bees can shed concentrations as high as 55,000 cells $/ \mu$ in their feces 
${ }^{39}$, suggesting flies can encounter and potentially ingest much higher $C$. bombi loads than the 3,200 cells we used for inoculum. We found that inoculated $E$. tenax flies defecated $C$. bombi in levels lower than those ingested. However, all $C$. bombi quantities found in the fly feces were capable of establishing an infection in bumble bees. Specifically, we show that an inoculation dosage of only $24 \mathrm{C}$. bombicells can establish an infection in bumble bees. This quantity of $C$. bombi is less than the lowest quantity we found in flower fly feces (60 cells) and much lower than the mean C. bombiquantity in flower fly feces (239 cells). As susceptible hosts range in size and immune-related traits, the number of parasites required to infect a smaller host, such as $M$. rotundata or O. lignaria, may be different compared to larger Bombus hosts. Therefore, the infectivity of $C$. bombiloads shown in this study may vary based on the susceptibility of host species.

While we report that $C$. bombi does not infect $E$. tenax flies, uninfected Eristalis flies still possess traits which increase their vectoring potential of the parasite by defecating frequently and in large volumes. $E$. arbustorum flies defecated comparable volumes to $O$. lignaria bees, and $E$. tenax flies defecated the largest fecal volumes of the four pollinators. Although both bees are competent vectors of $C$. bombi ${ }^{21}$, our results suggest that $O$. lignaria may be more likely to transmit the parasite, as this bee species defecates larger fecal volumes than $M$. rotundata. Susceptible pollinators are more likely to acquire fecalorally transmitted parasites from large volumes of infected feces when foraging, as large fecal events take longer to evaporate, thus allowing parasites to survive for a greater period of time outside of a host ${ }^{35}$. Differences in vectoring potential between competent hosts and non-host vectors warrant further investigation, with the possibility to reveal novel factors that may be incorporated into disease modelling of species-rich pollinator communities ${ }^{40}$.

We also demonstrated that bee and fly pollinators defecate on certain locations of a shared floral resource more frequently than others. The bee 0 . lignaria defecated the most on Solidago, which may suggest this known host of $C$. bombi spent the most amount of time on the floral resource compared to the two fly species. However, of the six locations (inside the corolla, outside the corolla, on the sepal, bract, stem and leaves), all three pollinators defecated most often on the inside of the corolla. Goldenrod is an important late-season resource for many pollinators, and since Crithidia is not a vertically transmitted parasite ${ }^{41}$, solitary pollinators foraging on flowers for pollen and nectar are likely acquiring the parasite at shared floral resources.

While $E$. tenax flies can act as mechanical vectors by ingesting and shedding viable $C$. bombicells, this does not necessarily imply an amplification in disease transmission. When vectors ingest parasites at a floral resource, they also remove them from existing floral hotspots; mechanical vectors redistribute parasites across the landscape by increasing the number of hotspots, but at the expense of decreasing the average parasite load per hotspot. Whether this redistribution leads to amplification from the increased number of hotspots, or a dilution from the decreased average load, depends on the doseresponse relationship between host and parasite and the number of non-host vectors capable of spreading the parasite ${ }^{42}$. The successful infection of bumble bees when fed low inoculum doses (e.g., 
only 24 cells) of $C$. bombi, however, suggest the potential for amplification, although ultimately this will need to be assessed using a quantitative epidemiological model. Furthermore, it is unknown how many

non-host vectors can spread bee parasites at flowers. Cook et al. ${ }^{43}$ found that flies from 86 families have been reported visiting the flowers of more than 1,100 different species of plants globally, however, flies are not the only insects sharing floral resources with bees.

When we assessed $C$. bombi viability in the feces of the $E$. tenax flies, we deemed the parasites viable if they were still motile. While the parasites were all actively swimming, we anecdotally observed that the parasites found in fly feces swam with less vigor compared to those that were harvested from bumble bee guts. Whether this impacts likelihood of infecting new hosts is unknown and beyond the scope of our current study. However, we suggest assessing the infectivity of Eristalis-defecated C. bombi for known hosts, such as Bombus spp., $O$. lignaria and $M$. rotundata bees. More broadly, little is known regarding the infectivity of mechanically transmitted fecal-oral parasites that pass through non-host vectors.

Our findings provide justification to look beyond bees to better understand the epidemiology of speciesrich pollinator communities. We show that while $E$. tenax flies are not hosts of $C$. bombi, they can defecate viable $C$. bombi cells. However, Eristalis flies still possess traits that facilitate the dispersal of fecal-orally transmitted "bee" parasites, by defecating frequently and in large volumes inside the corolla of flowers where susceptible hosts forage for nectar and pollen which has important implications not only for Crithidia parasite transmission networks, but general plant-pollinator-parasite networks. Also, our results suggest that the vectoring potential of known hosts of $C$. bombi may vary between species, as $M$. rotundata defecated significantly smaller fecal volumes than 0 . lignaria bees. Therefore, we recommend investigating whether more non-bee pollinators that share floral resources with bees can be hosts or nonhost vectors of bee parasites so these species can be incorporated in epidemiological models of pollinator communities.

\section{Methods}

\subsection{Rearing methodology}

Eristalis flower flies were reared in laboratory conditions from egg clutches laid by wild-caught females in the summer of 2019 (see Supplementary Materials for detailed rearing methodology). Only flies that emerged on the same day were used in the experiments. An artificial diapause protocol (see Supplementary Materials for detailed protocol) was used to prolong the lifespan of lab-reared flower flies, as adult Eristalis flower flies in lab colonies have shorter lifespans than adult Eristalis flies in the wild ${ }^{44}$. Once the adult flies eclosed, all siblings were placed in artificial diapause in a refrigerator and fed $10 \%$ sucrose ad libitum until the experiment began. These Eristalis flower fly rearing and artificial diapause protocols are a modification of previously published protocols ${ }^{44,45}$.

Osmia lignaria ( $n=50$; Crown Bees, Woodinville, WA, USA) and Megachile rotundata $(n=50$; Watts Solitary Bees, Bothell, WA, USA) were purchased and allowed to eclose in an incubator kept at 23 degrees 
C and $65 \%$ humidity. Bumble bees (Bombus impatiens) used as $\mathrm{C}$. bombi source colonies or as uninfected sources of bees for the dose-response trials were purchased from Biobest (Biobest, Leamington, Ontario, Canada) and maintained in the lab by feeding sucrose and pollen from a mixture of honey bee-collected poly-floral pollen (Bee Pollen Granules, CC Pollen High Desert, Phoenix AZ, USA).

\subsection{Evaluating whether the European drone fly, Eristalis tenax, is a host of Crithidia bombi}

After breaking artificial diapause, the E. tenax flower flies were allowed to groom, but not feed, for one hour. Each fly was then placed abdomen-first into a $1.5 \mathrm{~mL}$ microcentrifuge tube harness to collect defecation events (Supplementary Figure S5). The size of these tubes allowed the flies to feed comfortably, but the tubes were also tapered at the bottom, which prevented the flies from stepping in their feces. Holes were placed along the side of the tubes so the fly could respire. One large hole was placed on the lid of the tube so the fly could be inoculated directly with a pipette.

Flies were randomly divided into treatment and control groups. E. tenax flies in a roughly 1:2 F:M sex ratio were used in both the treatment $(n=30)$ and control groups $(n=30)$, for a total of 60 replicates. The flies that emerged from the same egg clutch with this 1:2 F:M sex ratio were the only siblings that could accommodate the replicates needed for this experiment, which is why this sex ratio was used.

The C. bombi inoculum was made fresh from infected B. impatens (Hymenoptera: Apidae) individuals the morning of the experiment using established protocols. Briefly, we dissected the gut of infected B. impatiens workers from a laboratory source colony that sustained a strain collected from wild B. impatiens workers from Massachusetts, USA (GPS coordinates: $42.363911 \mathrm{~N},-72.567747 \mathrm{~W}$ ). We homogenized the bee guts in distilled water and diluted the mixture to $1280 \mathrm{C}$. bombi cells $\mu^{-1}$, which we

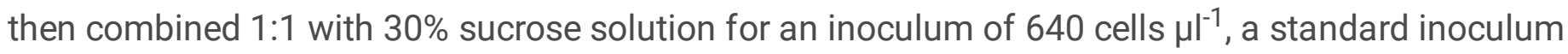
concentration for infecting bumble bees with $\mathrm{C}$. bombi ${ }^{35,46}$. Control groups were fed $5 \mu \mathrm{L}$ of a $30 \%$ sucrose and blue dye (Butler Extract Co., Lancaster, PA, USA) that in pilot experiments was not found to influence host or parasite survival. Treatment groups were inoculated with $5 \mu \mathrm{L}(3,200$ cells total) of $\mathrm{C}$. bombi, $30 \%$ sucrose and blue dye solution. The number of cells used in the inoculum is similar to levels of $\mathrm{C}$. bombi found in the feces of bumblebees with recently established infections ${ }^{37}$. Blue dye was used to better visualize when fecal events occurred and flies that did not drink the entire $5 \mu \mathrm{L}$ inoculum were not used in the experiment.

After feeding, the flies were monitored continuously until defecation occurred. As these flies recently emerged from artificial diapause and were starved pre-experiment, every hour post- inoculation the flies were fed a $30 \%$ sucrose and blue dye solution ad libitum to encourage defecation. Once a fly defecated, the feces were collected via pipette and diluted to a $10 \mu \mathrm{l}$ solution with deionized water to observe and count parasites using Kova Glasstic slides. The fly was then placed in an individual $60 \mathrm{~mL}$ plastic portion cup with filter paper (Sigma-Aldrich, St Louis, MO, USA) and a $1.5 \mathrm{~mL}$ microcentrifuge tube feeder containing $500 \mu \mathrm{l}$ of a $30 \%$ sucrose and blue dye solution for 10 days. Feeders and filter papers were 
replaced every three days to prevent mold growth. As C. bombi typically replicates in high numbers after 10 days in the guts of bumble bees ${ }^{47}$, both control and treatment flies were dissected and $\mathrm{C}$. bombi gut counts were performed 10 days post-inoculation. Since actively swimming, and thus live, C. bombi is infective to susceptible bumble bee hosts ${ }^{35}$, only actively swimming C. bombi were counted. The fecal volume, dilution factor and counts of $\mathrm{C}$. bombi were quantified for each individual fly to calculate the exact amount of C. bombi in the individual's first defecation event.

\subsection{Dose-response data}

Crithidia bombi inoculum was made from infected B. impatiens (Hymenoptera: Apidae) individuals the morning of each trial using the protocols described above, with two exceptions. First, the $\mathrm{C}$. bombi strain was collected from wild B. impatiens workers from New York, USA (GPS coordinates: 42.457350, -76.426907). Second, a range of serially diluted doses were used to inoculate uninfected B. impatiens workers. The doses were: 25,000 cells, 12,500 cells, 6,250 cells, 3,125 cells, 1,563 cells, 781 cells, 391 cells, 195 cells, 98 cells, 49 cells, 24 cells, and 12 cells. To obtain these doses, we homogenized bee guts in distilled water and diluted the mixture to $5,000 \mathrm{C}$. bombi cells $\mathrm{Hl}^{-1}$ with $30 \%$ sucrose solution. Serial dilutions were then conducted with a $10 \%$ sucrose solution to ensure the same osmolarity of each inoculum.

We conducted four replicate dose-response trials over a period of four weeks. Each week, 5 uninfected workers per dose from each of two colonies were administered $5 \mu$ of C. bombi inoculum. The ten highest doses were administered for the first two weeks, and two additional doses ( 24 cells, and 12 cells) were added for the final two weeks. Inoculated bees were kept individually in vials and fed $30 \%$ sucrose ad lib for seven days at 23 degrees $\mathrm{C}$ and $65 \%$ humidity. After seven days, the bees were dissected and $\mathrm{C}$. bombi loads were quantified using a hemocytometer as described above. In addition, the right forewing was removed from each bee and marginal cell length was measured as a proxy for size ${ }^{48}$. In total, 220 bees were inoculated (20 replicates for each of the ten highest doses, 10 replicates for the two lowest doses).2.4. Vectoring potential of two bee species, Osmia lignaria and Megachile rotundata, and two Eristalis fly species, E. arbustorum and E. tenax

\subsubsection{Defecation patterns on a shared floral resource}

All pollinators (O. lignaria, M. rotundata, E. arbustorum and E. tenax) were placed in individual $60 \mathrm{~mL}$ plastic portion cups lined with filter paper. Each pollinator received a $1.5 \mathrm{~mL}$ microcentrifuge tube feeder containing $500 \mu \mathrm{l}$ of fluorescent dye via $2.5 \mathrm{~g}$ of fluorescent powder (Stardust Micas) dissolved into 500 $\mathrm{mL} 30 \%$ sucrose feeders to visualize fecal deposition on flowers. After 24 hours, filter papers were collected (for analysis of fecal volume and defecation frequency, see below) and a total of five, randomly selected pollinators of the same species were placed in $12 \times 12 \times 12$ " mesh cages (Bioquip Products, Rancho Dominguez, CA, USA) containing inflorescences of similar sized Solidago "Dansolitlem" hybrida, little lemon goldenrod each replicate trial. Little lemon goldenrod was used in this experiment because both bees and flower flies were observed foraging on this abundant floral resource. Only pollinators with filter papers containing fluorescing defecation events were released in the mesh cages. 
All E. arbustorum cages $(n=10)$ contained 2:3 F:M sex ratios, except one cage contained a 3:2 F:M sex ratio. All E. tenax cages $(n=20)$ contained 3:2 F:M sex ratios, except four cages contained 2:3 F:M sex ratios. All O. lignaria cages $(n=10)$ contained 4:1 F:M sex ratios, except one cage contained a 3:2 F:M sex ratio. For the two fly species, sample sizes and F:M sex ratios were determined by the greatest, same-day sibling emergence. For $\mathrm{O}$. lignaria, sample sizes and $\mathrm{F}: \mathrm{M}$ sex ratios were determined by emergence availability. M. rotundata floral deposition data was not collected, as the F:M emergence was heavily skewed to males that did not interact with, and therefore defecate on, the flowers.

After 24-hours, the pollinators were removed and the defecation events on the goldenrod from all cages were counted under a blacklight. The location of the defecation events on the goldenrod was recorded. The plant parts were divided into six categories: 'inside' the flower (inside the corolla), 'outside' the flower (surface of the corolla), on the sepal, on the bract (the leaflike structure beneath the flower), on the stem or on a leaf.

\subsubsection{Defecation frequency and fecal volumes}

The diameter of the smallest and largest defecation events per filter paper was measured by a digital caliper and an average diameter was calculated from these two values for all pollinators. The average diameter of the defecation events was converted to an average volume (in $\mu l)$ using a standard curve Supplementary Figure S6). $\mathrm{R}^{2}=0.99$ for the calibration data). The collected fecal volumes defecated by control flies from the E. tenax inoculation experiment (see above) were compared to the average fly fecal volumes calculated here. This was done to analyze whether flies in a confined environment, where they were inoculated with $\mathrm{C}$. bombi, defecated similar volumes to flies allowed to move freely in an individual cup, which the average volumes were estimated from. In addition, the number of defecation events (frequency) over a 24-hour period on the collected E. arbustorum $(n=46)$ and E. tenax $(n=100)$ filter papers were counted for each fly.

\subsection{Statistical analyses}

For the E. tenax inoculation experiment, we evaluated the amount of C. bombi cells in the first defecation event using a negative binomial generalized linear model (GLM), with fly sex as predictor. We chose negative binomial over Poisson to account for overdispersion, which we evaluated using Pearson residuals. Significance of sex was evaluated using a likelihood ratio test (LRT).

Data from the B. impatiens inoculation experiment were used to fit two dose-response curves, the first for infection probability, and the second for infection intensity among infected bees. Infection intensity was defined using the loads estimated from the hemocytometer. A bee was considered infected if the counts were nonzero. We first tested whether the dose ingested, wing length (as a proxy for body size) and the colony the bee came from affected its response. For infection probability, this was done using a GLM with $\log _{10}$ (dose), colony, wing length and their interactions as predictors, and infection status as the Bernoulli response. For infection intensity, this was done using a linear model (LM) with the same predictors, and $\log _{10}$ (intensity) as response, using only infected bees. Doses were log-transformed in 
accordance to how the experimental doses were varied, while intensities were log-transformed to achieve normality of the residuals. Significance of predictors were tested in accordance with the principle of marginality.

While we found that wing length and colony were significant predictors, in practice the colony-specific response of a wild bee is unknown (since it would not have come from any of the experimental colonies), while the dependence on wing length is only useful in a size-based epidemiological model. Hence, we generated dose-response curves by marginalizing across colony and wing length. Finally, we tested whether linear relationships between the link function and $\log _{10}$ (dose), assumed in LMs and GLMs, were sufficient to capture the shape of the dose-response curves, by fitting the data to shape-constrained additive models and then comparing AIC values ${ }^{49}$. SCAMs are generalized additive models (GAMs) on which additional constraints such as monotonicity have been imposed; being more flexible, they can better capture the shapes of the dose-response curves should the linear relationships be inadequate.

We evaluated whether fecal volume depended on pollinator species and sex with a linear model (LM), fitted using weighted least squares to account for unequal variances between group (detected using Levene's test). Since the transformation from diameter (of feces on filter paper) to volume introduced a noticeable skew to the distribution, we transformed the volume back to diameter and further performed a Box-Cox transformation to achieve normality ${ }^{50}$, which we verified using the Shapiro-Wilk and D'Agostino's $\mathrm{K}^{2}$ test. The transformed volume was used as the response in the abovementioned linear model.

For E. tenax, fecal volume was also manually collected from the 1.5 microcentrifuge tubes during the inoculations experiment. We compared the fecal volume from the two methods using a LM with method and fly sex as well as their interaction as predictors. Volumes were log-transformed to achieve normality, while the linear model was fitted using ordinary least squares since Levene's test indicated no significant deviation from the assumption of equal variance.

We evaluated whether defecation frequency depended on pollinator species and sex with a LM, again fitted using weighted least squares to account for unequal variances between groups. While two of the groups showed deviation from normality using the Shapiro-Wilk test, the deviations were only marginally significant and hence not expected to qualitatively affect the results ${ }^{51}$.

Finally, we evaluated defecation patterns on Solidago using a negative binomial GLM, with feces counts as the response, and pollinator species, plant location and their interaction as predictors. We did not use a mixed model with cage number as a random effect since there was only one count value per cage per location, so pseudo-replication was not an issue. Significance of predictors were evaluated using LRT in accordance to the principle of marginality ${ }^{52}$ (i.e., main effects were tested only when their interactions were insignificant and hence dropped). Post-hoc tests of pairwise contrasts with Tukey corrections were performed for predictors that were significant. We recognize that the principle sex ratio and its interactions with other predictors could also be included among the predictors; however, since each 
species had cages with predominantly one sex ratio (E. tenax 3F:2M; E. arbustorum 2F:3M; O. lignaria mix of $4 \mathrm{~F}: 1 \mathrm{M}$ and $5 \mathrm{~F}: 0 \mathrm{M})$, this meant that species and sex ratios were highly correlated, making it impossible to separate their effects. Nonetheless, since female Eristalis flies do not provision their brood, the differences between sexes (e.g., time spent foraging on plants) may be less pronounced than in bees.

\section{Declarations}

\section{Acknowledgements}

We thank Laura Figueroa and Julie Davis for helpful advice regarding methodology. Leeah Richardson, Zoe Kim, Steven Wang and Brandon Woo for help with collections of wild Eristalis adults to start a colony, and Cali Grincavitch helped with Eristalis fly colony care.

\section{Author contributions}

A.E.D., K. R. D., W. H. N. and S. H. M. conceived and/or designed the experiment A. E. D., A. M. T., M. J. M., L. C., E. H., D. S. and P. A. M. collected the data. A.E.D., W. H. N. and S. H. M. analyzed and/or interpreted the data. A. E. D. drafted the manuscript. All authors revised the manuscript.

\section{Competing Interests}

The author(s) declare no competing interests.

\section{Funding}

This research was funded by the National Institute of General Medical Sciences of the National Institutes of Health [grant number R01GM122062], the alumni of the Cornell University Chapter of Alpha Zeta, CALS Charitable Trust [grant number 1718], and the Cornell University Agricultural Experiment Station, CALS Hatch supplement [grant number 1819]. The content is solely the responsibility of the authors and does not necessarily represent the official views of the National Institutes of Health

\section{References}

1. Biesmeijer, J. C. et al. Parallel declines in pollinators and insect-pollinated plants in Britain and the Netherlands. Science. 313, 351-354 https://doi.org/10.1126/science.1127863 (2006).

2. Potts, S. G. et al. Global pollinator declines: trends, impacts and drivers. Trends Ecol Evol. 25, 345353 https://doi.org/10.1016/j.tree.2010.01.007 (2010).

3. Hallmann, C. A. et al. More than 75 percent decline over 27 years in total flying insect biomass in protected areas. Plos One 12, doi:ARTNe018580910.1371/journal.pone.0185809(2017). 
4. Powney, G. D. et al. Widespread losses of pollinating insects in Britain. Nat Commun 10, doi:ARTN 101810.1038/s41467-019-08974-9(2019).

5. Cameron, S. A. et al. Patterns of widespread decline in North American bumble bees. P Natl Acad Sci USA. 108, 662-667 https://doi.org/10.1073/pnas.1014743108 (2011).

6. Schmid-Hempel, R. et al. The invasion of southern South America by imported bumblebees and associated parasites. J Anim Ecol. 83, 823-837 https://doi.org/10.1111/1365-2656.12185 (2014).

7. Goulson, D., Nicholls, E., Botias, C. \& Rotheray, E. L. Bee declines driven by combined stress from parasites, pesticides, and lack of flowers. Science 347, doi:ARTN125595710.1126/science.1255957(2015).

8. Klein, A. M. et al. Importance of pollinators in changing landscapes for world crops. P Roy Soc B-Biol Sci. 274, 303-313 https://doi.org/10.1098/rspb.2006.3721 (2007).

9. Kleijn, D. et al. Delivery of crop pollination services is an insufficient argument for wild pollinator conservation. Nat Commun 6, doi:ARTN 741410.1038/ncomms8414(2015).

10. Rader, R. et al. Non-bee insects are important contributors to global crop pollination. P Natl Acad Sci USA. 113, 146-151 https://doi.org/10.1073/pnas.1517092112 (2016).

11. Rader, R., Cunningham, S. A., Howlett, B. G. \& Inouye, D. W. Non-Bee Insects as Visitors and Pollinators of Crops: Biology, Ecology, and Management. Annu Rev Entomol. 65, 391-407 https://doi.org/10.1146/annurev-ento-011019-025055 (2020).

12. Reilly, J. R. et al. Crop production in the USA is frequently limited by a lack of pollinators. P Roy SoC B-Bio/ Sci 287, doi:ARTN 2020092210.1098/rspb. 2020.0922 (2020).

13. Aizen, M. A., Garibaldi, L. A., Cunningham, S. A. \& Klein, A. M. Long-Term Global Trends in Crop Yield and Production Reveal No Current Pollination Shortage but Increasing Pollinator Dependency. Curr Biol. 18, 1572-1575 https://doi.org/10.1016/j.cub.2008.08.066 (2008).

14. Schmid-Hempel, P. Mating, parasites and other trials of life in social insects. Microbes Infect. 2, 515520 https://doi.org/10.1016/S1286-4579(00)00316-6 (2000).

15. Graystock, P. et al. Dominant bee species and floral abundance drive parasite temporal dynamics in plant-pollinator communities. Nat Ecol Evol. 4, 1358-1358 https://doi.org/10.1038/s41559-0201247-x (2020).

16. Schoonvaere, K., Smagghe, G., Francis, F. \& de Graaf, D. C. Study of the Metatranscriptome of Eight Social and Solitary Wild Bee Species Reveals Novel Viruses and Bee Parasites. Front Microbio/ 9 , doi:ARTN 17710.3389/fmicb. 2018.00177 (2018).

17. Murray, E. A. et al. Viral transmission in honey bees and native bees, supported by a global black queen cell virus phylogeny. Environ Microbiol. 21, 972-983 https://doi.org/10.1111/14622920.14501 (2019).

18. Alger, S. A., Burnham, P. A., Boncristiani, H. F. \& Brody, A. K. RNA virus spillover from managed honeybees (Apis mellifera) to wild bumblebees (Bombus spp.). Plos One 14, doi:ARTN e021782210.1371/journal.pone.0217822(2019). 
19. Bramke, K., Muller, U., McMahon, D. P. \& Rolff, J. Exposure of Larvae of the Solitary Bee Osmia bicornis to the Honey Bee Pathogen Nosema ceranae Affects Life History.Insects10, doi:ARTN 38010.3390/insects10110380 (2019).

20. Tapia-Gonzalez, J. M. et al. Evidence of presence and replication of honey bee viruses among wild bee pollinators in subtropical environments. J Invertebr Pathol 168, doi:ARTN 10725610.1016/j.jip. 2019.107256 (2019).

21. Figueroa, L. L., Grincavitch, C. \& McArt, S. H. Crithidia bombi can infect two solitary bee species while host survivorship depends on diet. Parasitology. 148, 435-442 https://doi.org/10.1017/S0031182020002218 (2021).

22. Ngor, L. et al. Cross-infectivity of honey and bumble bee-associated parasites across three bee families.Parasitology147,1290-1304, doi:Pii S00311820200010181017/S0031182020001018 (2020).

23. Evison, S. E. F. et al. Pervasiveness of Parasites in Pollinators. Plos One 7, doi:ARTN e3064110.1371/journal.pone.0030641(2012).

24. Bailes, E. J. et al. First detection of bee viruses in hoverfly (syrphid) pollinators. Biol Letters 14, doi:ARTN 2018000110.1098/rsbl. 2018.0001 (2018).

25. Brettell, L. E., Riegler, M., O'Brien, C. \& Cook, J. M. Occurrence of honey bee-associated pathogens in Varroa-free pollinator communities. J Invertebr Pathol 171, doi:ARTN 10734410.1016/j.jip 2020.107344 (2020).

26. Lipa, J. J., Triggiani, O. \& Crithidia-Bombi Sp, N. A Flagellated Parasite of a Bumblebee BombusTerrestris L (Hymenoptera, Apidae). Acta Protozool. 27, 287-287 (1988).

27. Higes, M. et al. How natural infection by Nosema ceranae causes honeybee colony collapse. Environ Microbiol. 10, 2659-2669 https://doi.org/10.1111/j.1462-2920.2008.01687.x (2008).

28. Fries, I. Nosema ceranae in European honey bees (Apis mellifera). J Invertebr Pathol. 103, S73-S79 https://doi.org/10.1016/j.jip.2009.06.017 (2010).

29. Larson, B. M. H., Kevan, P. G. \& Inouye, D. W. Flies and flowers: taxonomic diversity of anthophiles and pollinators. Can Entomol. 133, 439-465 https://doi.org/10.4039/Ent133439-4 (2001).

30. Skevington, J. et al. Field guide to the flower flies of northeastern North America (Princeton University Press, 2019).

31. Graystock, P., Goulson, D. \& Hughes, W. O. H. Parasites in bloom: flowers aid dispersal and transmission of pollinator parasites within and between bee species. P Roy Soc B-Biol Sci 282, doi:ARTN 2015137110.1098/rspb 2015.1371 (2015).

32. Figueroa, L. L. et al. Landscape simplification shapes pathogen prevalence in plant-pollinator networks. Ecol Lett. 23, 1212-1222 https://doi.org/10.1111/ele.13521 (2020).

33. McArt, S. H., Koch, H., Irwin, R. E. \& Adler, L. S. Arranging the bouquet of disease: floral traits and the transmission of plant and animal pathogens. Ecol Lett. 17, 624-636 https://doi.org/10.1111/ele.12257 (2014). 
34. Adler, L. S., Irwin, R. E., McArt, S. H. \& Vannette, R. L. Floral traits affecting the transmission of beneficial and pathogenic pollinator-associated microbes. Current Opinion in Insect Science. 44, 1-7 (2021).

35. Figueroa, L. L. et al. Bee pathogen transmission dynamics: deposition, persistence and acquisition on flowers. Proceedings of the Royal Society B-Biological Sciences 286, 20190603(2019).

36. Adler, L. S. et al. Disease where you dine: plant species and floral traits associated with pathogen transmission in bumble bees. Ecology. 99, 2535-2545 https://doi.org/10.1002/ecy.2503 (2018).

37. Schmid-Hempel, P. \& Schmid-Hempel, R. Transmission of a pathogen in Bombus terrestris, with a note on division of labour in social insects. Behavioral Ecology and Sociobiology. 33, 319-327 (1993).

38. Otterstatter, M. C. \& Thomson, J. D. Within-host dynamics of an intestinal pathogen of bumble bees. Parasitology. 133, 749-761 https://doi.org/10.1017/S003118200600120x (2006).

39. Logan, A., Ruiz-Gonzalez, M. X. \& Brown, M. J. F. The impact of host starvation on parasite development and population dynamics in an intestinal trypanosome parasite of bumble bees. Parasitology. 130, 637-642 https://doi.org/10.1017/S0031182005007304 (2005).

40. Truitt, L. L., McArt, S. H., Vaughn, A. H. \& Ellner, S. P. Trait-Based Modeling of Multihost Pathogen Transmission: Plant-Pollinator Networks. Am Nat. 193, E149-E167 https://doi.org/10.1086/702959 (2019).

41. Schmid-Hempel, P. \& Loosli, R. A contribution to the knowledge of Nosema infections in bumble bees, Bombus spp. Apidologie. 29, 525-535 https://doi.org/10.1051/apido:19980605 (1998).

42. Ng, W. H., McArt, S. H., Myers, C. R. \& Ellner, S. P. Mechanical vectors amplify or dilute disease transmission depending on the host dose-response relationship.Manuscript submitted for publication(2021).

43. Cook, D. F. et al. The Role of Flies as Pollinators of Horticultural Crops: An Australian Case Study with Worldwide Relevance.Insects11, doi:ARTN 3413390/insects11060341 (2020).

44. Nicholas, S., Thyselius, M., Holden, M. \& Nordstrom, K. Rearing and Long-Term Maintenance of Eristalis tenax Hoverflies for Research Studies.Jove-J Vis Exp, doi:ARTN e5771110.3791/57711 (2018).

45. Ottenheim, M. M. \& Holloway, G. J. The effect of diet and light on larval and pupal development of laboratory-reared Eristalis arbustorum (Diptera: Syrphidae). Neth J Zool. 45, 305-314 (1995).

46. Richardson, L. L. et al. Secondary metabolites in floral nectar reduce parasite infections in bumblebees. Proc. R. Soc. B 282, 8(2015).

47. Schluns, H., Sadd, B. M., Schmid-Hempel, P. \& Crozier, R. H. Infection with the trypanosome Crithidia bombi and expression of immune-related genes in the bumblebee Bombus terrestris. Dev Comp Immunol. 34, 705-709 https://doi.org/10.1016/j.dci.2010.02.002 (2010).

48. Harder, L. D. Measurement and estimation of functional proboscis length in bumblebees (Hymenoptera: Apidae). Canadian Journal of Zoology. 60, 1073-1079 (1982). 
49. Pya, N. \& Wood, S. N. Shape constrained additive models. Stat Comput. 25, 543-559 https://doi.org/10.1007/s11222-013-9448-7 (2015).

50. Box, G. E. P. \& Cox, D. R. An Analysis of Transformations. Journal of the Royal Statistical Society. Series B (Methodological). 26, 211-252 (1964).

51. Glass, G. V., Peckham, P. D. \& Sanders, J. R. Consequences of Failure to Meet Assumptions Underlying the Fixed Effects Analyses of Variance and Covariance. Review of Educational Research. 42, 237-288 (1972).

52. Nelder, J. A. A Reformulation of Linear Models. Journal of the Royal Statistical Society. Series A (General). 140, 48-77 (1977).

\section{Figures}

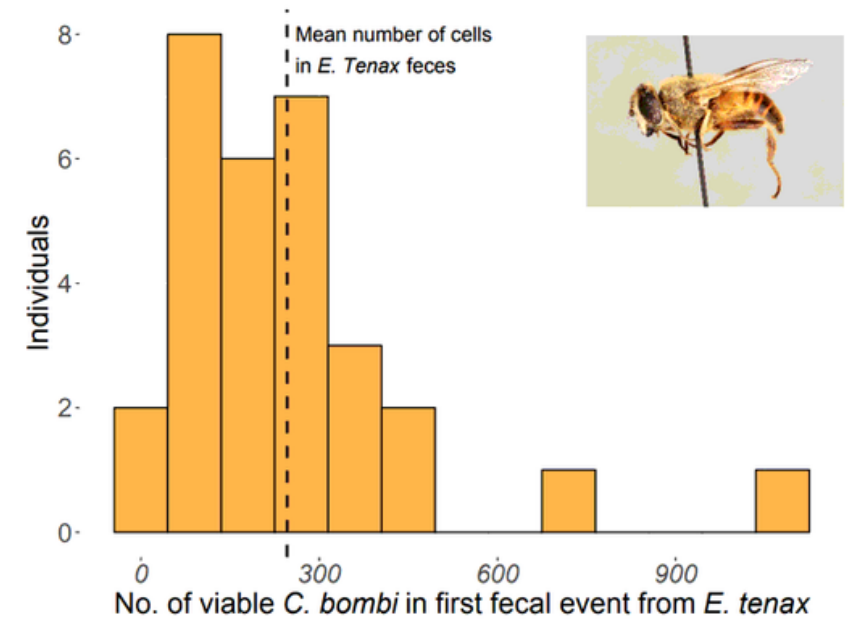

(a)

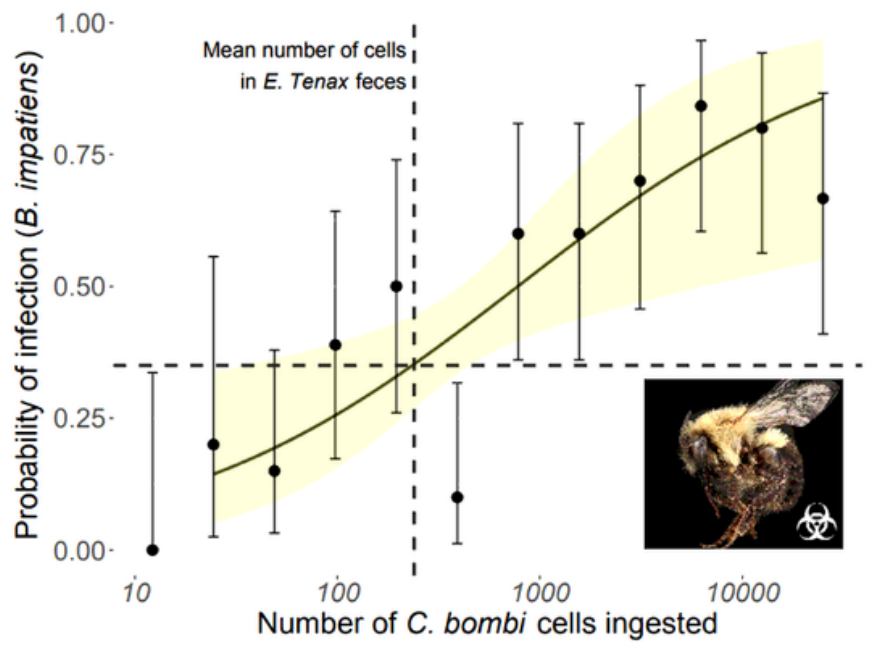

(b)

\section{Figure 1}

Flower flies can defecate enough live Crithidia bombi cells to infect bumble bee hosts: a) Total number of C. bombi cells found in the first fecal events of inoculated Eristalis tenax flies $(n=30)$; (b) Probability of C. bombi infection of inoculated Bombus impatiens bumble bees ( $n=9-12$ per dose). In both figures, the vertical dotted lines indicate the mean number of C. bombi (239 cells) defecated in the first fecal events of E. tenax flies. In figure (b), the horizontal dotted line indicates that the probability of infection at 239 cells is 35\%. 95\% confidence intervals are shown as error bars for each treatment and the shaded area on the dose-response curve. "Side view of Eristalis tenax" image by Ken Perry, licensed under CC0 1.0. "Side view of Bombus impatiens" image by Christopher Johnson, licensed under CC0 1.0. 


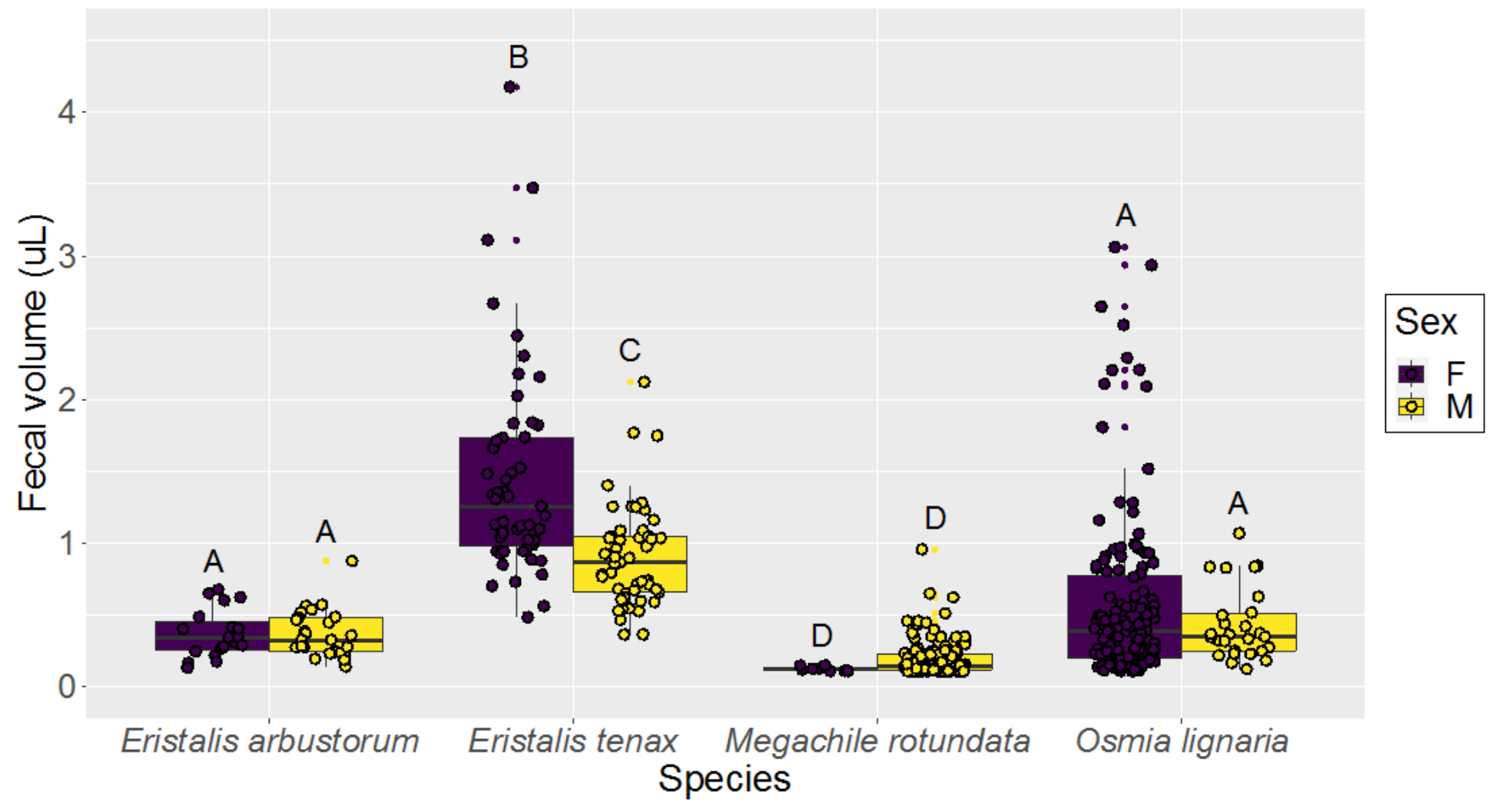

Figure 2

Fecal volumes of two flower fly pollinators, Eristalis arbustorum and E. tenax, and two bee pollinators, Megachile rotundata and Osmia lignaria. Whiskers indicate the range of fecal volumes, excluding outliers. Upper, middle and lower quartiles indicate the greatest, average and lowest fecal volumes collected, respectively. Data points have been jittered for clarity. Different letters indicate significant pairwise post-hoc contrasts $(p<0.05)$. 


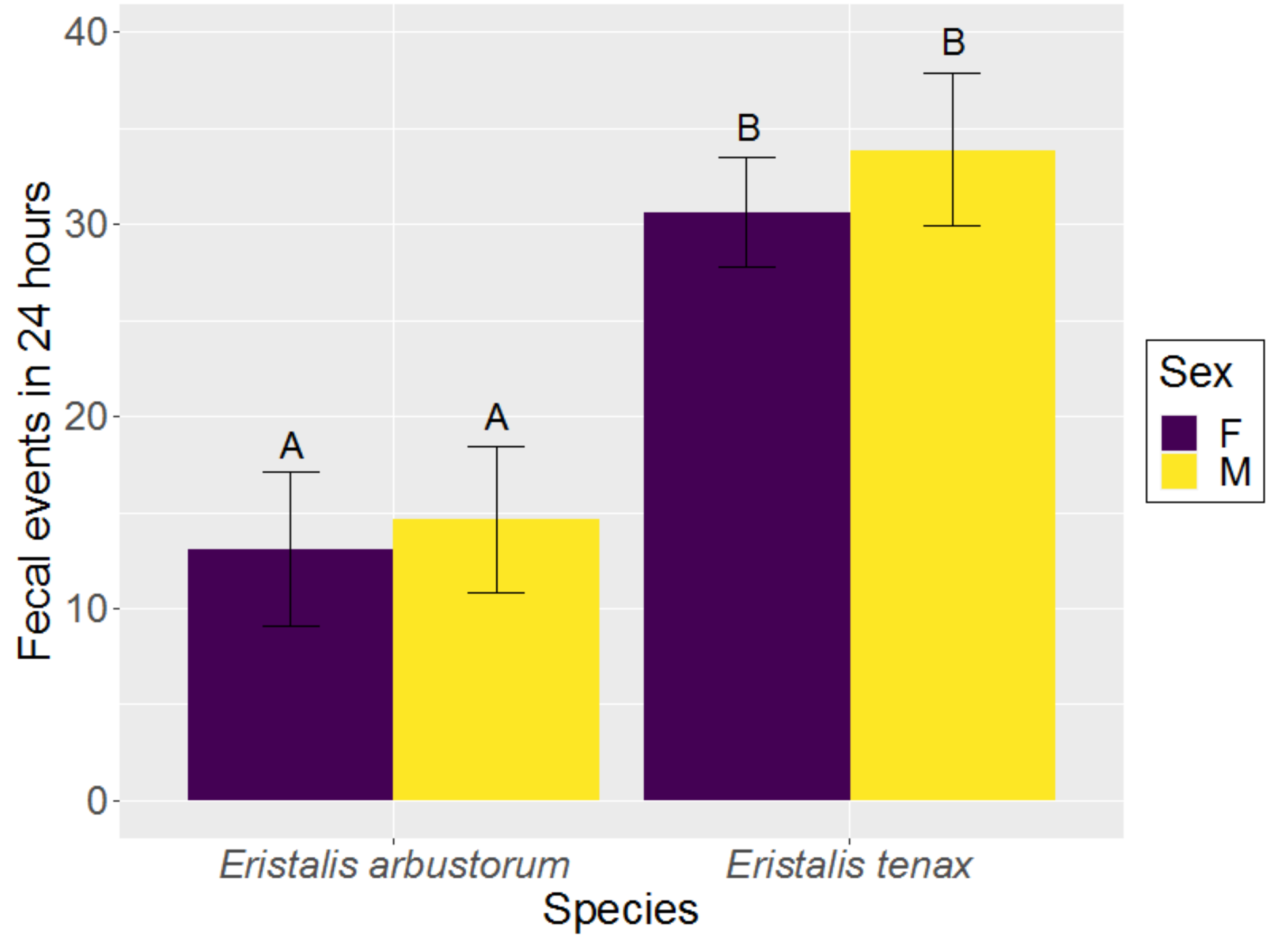

Figure 3

Number of fecal events by Eristalis arbustorum and E. tenax flies over 24 hours. Different letters indicate post-hoc significance $(p<0.05)$, error bars indicate $95 \%$ confidence intervals. 


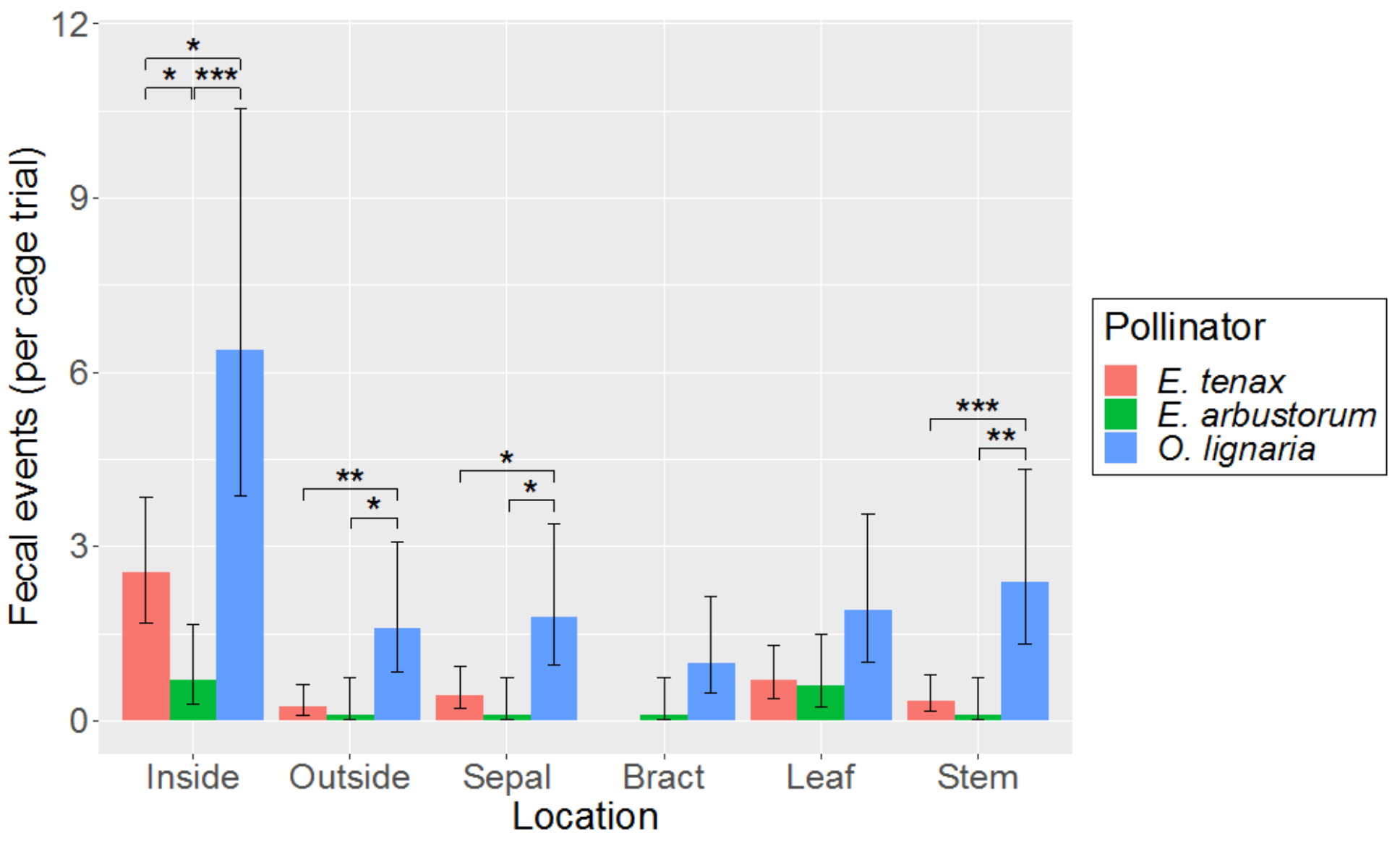

\section{Figure 4}

Number of Eristalis tenax, Eristalis arbustorum and Osmia lignaria fecal events per cage trial $(n=10$ each) at six different floral locations (inside the corolla, outside the corolla, on the sepal, bract, leaf or stem) of Solidago "Dansolitum" hybrida. Asterisks indicate Tukey-corrected significance of pairwise contrasts between species within each location ( ${ }^{\star \star *} p<0.001$; $\left.{ }^{*} p<0.01 ; * p<0.05\right)$. Error bars indicate $95 \%$ confidence intervals.

\section{Supplementary Files}

This is a list of supplementary files associated with this preprint. Click to download.

- DavisSupplementarymaterial.docx 